\title{
Intensified rehabilitation therapy and transitions to skilled nursing facilities in community-living seniors with acute medical illnesses
}

\author{
Ji Won Yoo, ${ }^{1,2}$ Sulgi Kim, ${ }^{3}$ Jai Hyun Choi ${ }^{4}$ and Woo Sang Ryu ${ }^{5}$ \\ ${ }^{1}$ Department of Internal Medicine, University of Michigan Medical School, ${ }^{2}$ Geriatric Research, Education and Clinical Center (GRECC), \\ Veterans Affairs Ann Arbor Healthcare System, Ann Arbor, Michigan, ${ }^{3}$ Department of Epidemiology, School of Public Health, University \\ of Washington, Seattle, Washington, USA, and Departments of ${ }^{4}$ Internal Medicine and ${ }^{5}$ Surgery, Korea University, Seoul, Korea
}

Aim: To examine whether rehabilitation therapy type would be associated with transitions to skilled nursing facilities (SNF) in community-living seniors with acute medical illnesses.

Methods: Using administrative and clinical data, multivariate regression analysis examined the relationship between the extent of rehabilitation therapy and transitions to SNF in all participants, as well as participants by physical function at admission.

Results: In all participants $(n=929)$, the intensified rehabilitation therapy was associated with a lower probability of transitions to SNF ( $14 \%$ vs $21 \%$; odds ratio [OR] 0.59 ; $95 \%$ confidence intervals $[\mathrm{CI}] 0.22-0.96 ; P=0.02)$. In participants with mild physical limitations $(n=270)$, less frequent transitions to SNF occurred when patients received intensified rehabilitation therapy [ $16 \%$ vs $23 \%$; OR 0.46 ; $95 \%$ CI $0.17-0.94 ; P=0.01]$. In participants with moderate to severe physical limitations $(n=265)$, the decreased frequency of transitions to SNF associated with rehabilitation therapy became more pronounced $(18 \%$ vs $28 \%$; OR $0.34 ; 95 \%$ CI $0.07-0.89 ; P=0.004)$. By contrast, in participants without physical limitation $(n=394)$, the number of transitions to SNF did not change significantly when they received intensified rehabilitation therapy $(P=0.53)$.

Conclusions: We found a significant relationship between intensified rehabilitation therapy and the decrease of transitions to SNF in community-living seniors with acute medical illness. The magnitude of this relationship increased in participants with more physical limitations, but not in participants without physical limitations at admission. Geriatr Gerontol Int 2013; 13: 547-554.

Keywords: frail older adults, geriatric medicine, hospital units, rehabilitation outcome, skilled nursing facility.

\section{Introduction}

More than three-quarters of nursing home admissions in the USA are preceded by an acute care hospitalization in the prior 120 days. ${ }^{1}$ Previous studies provide evidence that acute illnesses requiring hospitalization result in catastrophic or progressive disability in older adults. ${ }^{2,3}$ Furthermore, older adults recently disabled as a result of acute illness are vulnerable to nursing home placement regardless of the level of physical activity before hospitalization. ${ }^{4-6}$ Numerous studies have found that

Accepted for publication 19 July 2012.

Correspondence: Dr Ji Won Yoo MD MSc, Department of Internal Medicine, University of Michigan Medical School, 300 North Ingalls Building, Room 932, Ann Arbor, MI 48109, USA. Email: yoojiw@trinity-health.org more than one-third of hospitalized older adults experience decrements in physical function and more than one-quarter of them experience the transition to a nursing home. ${ }^{7,8}$

A skilled nursing facility (SNF) is a gateway for community-dwelling older adults to enter a nursing home setting. Transitions from hospital to SNF account for more than three-quarters of the nursing home entries by community-living seniors. ${ }^{5,9-11}$ Once admitted to a nursing home, the length of stay can be difficult to predict. For both hospitalized older adults and their families, preventing or deferring nursing home entries hold substantial implications for their psychosocial stability; thus, it might promote their quality of life to defer, as found in prior health survey studies. ${ }^{12,13}$

Rehabilitation therapy for older adults has been examined as a possible preventive measure to reduce 
the progress of physical disability in older adults at postacute or intermediate care settings across diverse health systems. ${ }^{8,14-16}$ A number of previous studies examined the impact of a geriatric rehabilitation unit (also known as a geriatric evaluation and management [GEM] unit) in post-acute care on restoring or preventing decline of physical function. ${ }^{17-20}$ These studies identified the dosedependent benefits of rehabilitation therapy: restoring physical function and reducing nursing home admission. ${ }^{21,22}$ The strongest evidence of improving the hospital outcomes of older adults, including the reductions in hospital stays and nursing home admissions, stems from geriatric inpatient units (acute care of elders [ACE] or post-acute care [PAC] units). ${ }^{23-28}$ Either ACE or PAC units are interdisciplinary care units for frail older adults with protocol for prevention of disability. In contrast to $\mathrm{ACE}$ and GEM units, the inpatient geriatric consultative model has failed to show an improvement of the same outcomes in hospitalized older adults..$^{29,30}$ Isolated geriatric assessment and management service without intensified rehabilitation therapy was reported to be less effective than an integrative care model including intensified rehabilitation as a core health service. ${ }^{25-29}$

The USA hospital Medicare margin has been negative since 2003. In addition, since the collapse of the USA bond and stock markets in 2008, operating hospital margins fell dramatically. ${ }^{31-33}$ Financial pressure on USA hospitals is expected to be more remarkable than ever before. The Patient Protection and Affordable Care Act (PPACA) attempts to cut current hospital operating margin deficits by reducing hospital length of stay, thus meeting diagnosis-related group (DRG) standards. ${ }^{32,33}$ This situation might have facilitated the rapid growth in use of SNF after hospital discharge. . $32,34^{2}$ Therefore, hiring a geriatric care specialist or establishing a geriatric unit or team, either of which would require significant financial investment, might meet with resistance from those who manage hospital budgets.

Yet, relatively little is known about the outcomes of intensified rehabilitation therapy for older adults during their acute hospital stays. Despite the high transition rate to SNF for this population, to our knowledge, no prior study has evaluated the potential role of intensified rehabilitation therapy for community-living seniors with acute medical illness to impede or precipitate these transitions. ${ }^{1,32,34}$ A lack of studies might possibly stem from the assumption that rehabilitation therapy for older adults with acute medical illness might be challenged by the patients' unstable medical conditions and busy schedules for frequent diagnostic/therapeutic procedures. ${ }^{7,8,17,18}$ The present study determines the relationship between rehabilitation therapy type (intensified vs usual) and transitions to SNF in community-living seniors with acute medical illness. We also examine how the effects of physical function at admission on this relationship vary.

\section{Methods}

\section{Study design and data collection}

The study sites were two USA metropolitan teaching hospitals with 350 (hospital A) and 415 (hospital B) beds. Study site hospitals have neither a geriatric unit nor geriatric consultation service teams. The study period was 2 years (January 2009 to December 2010). Inclusion criteria were: (i) patients were Medicare beneficiaries; (ii) aged 65 years or older; (c) admitted to the general medicine floor; and (d) community-living persons before hospital admission. Exclusion criteria were: (i) enrolment in hospice care or admission to an intensive care unit; (b) "extreme" severity of illness at admission per the All Patient Refined Diagnostic Related Group (APR-DRG), because these participants are not eligible to receive rehabilitation therapy during their hospitalizations; and (c) admission to the stroke service unit where rehabilitation therapy had been provided separately from the general medicine floor.

Administrative claim data were collected from the Department of Medical Operations, which was unaware of the study objectives and was abstracted by the researchers. Clinical data from electronic medical records were matched with administrative data by name and date of birth. For internal consistency and validation of extraction accuracy, a random sample of $10 \%$ of matched data was cross-reviewed. We estimated that a sample size of 462 was required for each group (usual and intensified rehabilitation therapy) based on an expected decrease in SNF transition rate from 21\% (usual rehabilitation) to $14 \%$ (intensified rehabilitation) with an alpha of $5 \%$, power of $80 \%$ and a two-sided analysis. ${ }^{1,5,6,35}$ We increased the sample size by $20 \%$, to a total of 554 patients per group, to account for possible follow-up loss. Figure 1 shows the flow chart of sample enrolment, allocation, follow up and analysis. The initial participants meeting inclusion criteria were 1108 . A total of 101 participants were excluded for the following reasons: hospice or intensive care enrolment $(n=12)$, "extreme" severity of illness $(n=36)$ and admission to the stroke service unit $(n=53)$. Among participants allocated to the intensified rehabilitation group $(n=501), 29$ participants were withdrawn because of placement at other destinations $(n=19)$ and death $(n=9)$. Among participants allocated to the usual rehabilitation group $(n=506), 30$ participants were withdrawn because of placement at other destinations $(n=17)$ and deaths $(n=13)$. Other destinations included long-term residential facilities and referrals to other hospitals. Long-term residential facilities included residential care homes, assisted living facilities, group homes and adult foster homes where rehabilitation therapy was not usually provided. Referrals to other hospitals included substance abuse care and psychiatric 
Figure 1 Flowchart of sample enrolment, allocation, follow up and analysis.

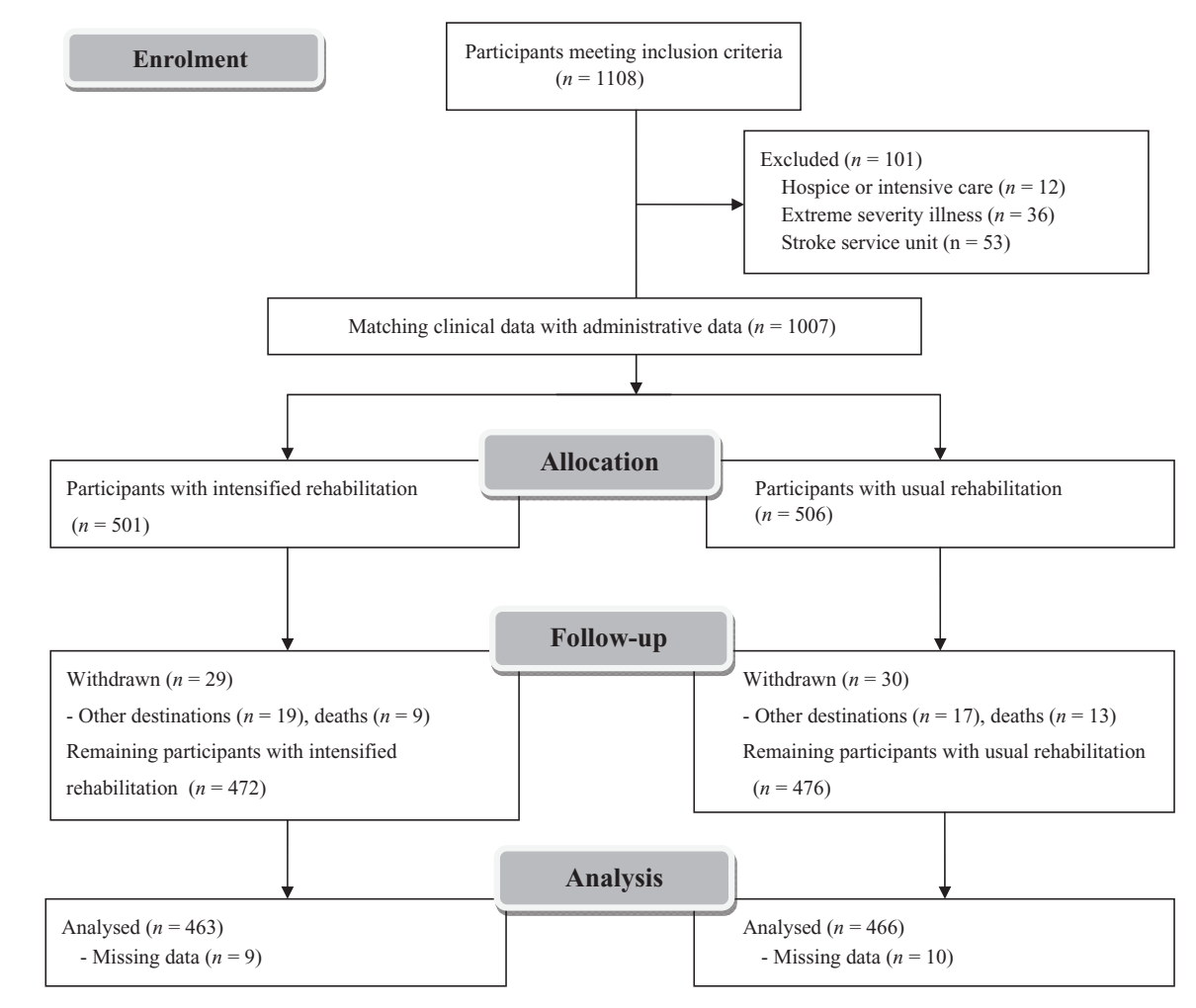

hospitals. Missing data occurred in nine instances in the intensified rehabilitation group and 10 instances in the usual rehabilitation group. The final number of analyzed participants was 463 in the intensified rehabilitation group and 466 in the usual rehabilitation group.

\section{Main outcomes: Discharge places}

Discharge places were categorized into either "SNF" or "return to their communities". We defined the main outcome as transitions from acute care hospital to SNF. SNF included extended-care facilities, where rehabilitation therapy was provided as a core medical service. "Return to their communities" meant discharge to home, where seniors had lived before hospital admission.

\section{Covariates}

Covariates were age, sex, ethnicity, living situation, severity of illness, study site, physical function at admission, rehabilitation therapy, cognitive impairment, delirium and principal diagnoses at admission. Living situation was categorized as either "living at risk" or "secure and stable living". "Living at risk" was defined as either living alone or in need of home care or day care services before hospital admission. Participants in need of social care services (either home care or day care services) without receiving these services were categorized as "living at risk". Participants who received these services were categorized as "secure and stable living". Participants not meeting the criteria for "living at risk" were defined as "secure and stable living". ${ }^{36}$

The APR-DRG severity of illness classification system was used to estimate the severity of illness. ${ }^{37}$ The APRDRG data were gathered based on the $3 \mathrm{M}$ Health Information System (Wallingford, CT, USA). The APR-DRG data were collected from the Department of Medical Operations, which was unaware of this study's objectives and was abstracted by the authors. The validity of APR-DRG severity of illness in acute hospital care has been discussed elsewhere. ${ }^{35}$

Study sites were hospitals A and B. To define rehabilitation therapy, we examined the length of stay (LOS) across all relevant therapies added together. For example, we added the total physical therapy (PT) and occupational therapy (OT) hours and divided the sum by the LOS. ${ }^{21,22}$ Patients did not need to receive both therapies to be included in these analyses. We defined $0.5 \mathrm{~h}$ or more a day as rehabilitation therapy. Patients did not need to receive all therapies to be included in these analyses. To compare the prior studies, we defined $0.5 \mathrm{~h}$ or more a day as rehabilitation therapy. ${ }^{21,22}$

Physical function was also assessed by admission nursing staff. The Katz Index of Independence in Activities in Daily Living (ADL) was the assessment tool of physical function with a range from most dependent (0) to most independent (6). ${ }^{38}$ The Katz Index has shown to have an acceptable internal consistency $(\alpha=0.87)$ and validity ( $\kappa=0.74$ to 0.88 ) when nurses carried out the 
assessment. $^{39,40}$ The interobserver agreement $(\kappa=0.64)$ and intraobserver reproducibility $(\kappa=0.88)$ of the Katz Index of the present study were relatively high. The Katz Index was categorized by the severity of physical limitation as follows: no limitation (6), mild limitations (4-5) and moderate to severe limitations $(0-3) .{ }^{6}$ Validating the categorization of the Katz Index for hospitalized older adults has been addressed elsewhere. ${ }^{6}$

Information on cognitive impairment before hospital admission was collected from medical records by researchers, and included Alzheimer's disease, vascular dementia, dementia with Lewy bodies, dementia with Parkinsonism, Pick's disease, senile degeneration of the brain, cerebral degeneration, uncomplicated senile dementia, dementia not otherwise specified and cognitive disorder not otherwise specified. The Confusion Assessment Method (CAM) was used to detect the development of delirium. It has a sensitivity between $94 \%$ and $100 \%$, and a specificity of $90-95 \%$, with high interobserver reliability. ${ }^{41}$ Delirium was assessed daily from hospital admission by physicians. Principal diagnoses at admission were classified into the following eight groups: (i) cardiovascular diseases; (ii) respiratory diseases; (iii) cancer and blood organ diseases; (iv) infectious diseases; (v) endocrine and metabolic diseases; (vi) digestive and urogenital tract diseases; (vii) neurological diseases except for acute stroke; and (viii) others. Classification of diseases and procedures was based on the International Classification of Diseases, Ninth Revision, Clinical Modification (ICD-9-CM).

For ease of interpretation, participant characteristics were categorized as follows: ethnicity (white people vs non-white people), living situation (living at risk vs secure and stable living), severity of illness (mild vs moderate vs major), study site (hospital A vs B), rehabilitation therapy (intensified vs usual), physical limitations at admission (none vs mild vs moderate-severe limitations), cognitive impairment (yes vs no), delirium (yes vs no) and principal diagnoses at admission (8 groups). ${ }^{35}$ The study was approved by the institutional review board at Cleveland Clinic Health System. Because data were not directly gathered from patients, the requirement to obtain informed consents from patients was waived.

\section{Statistical analysis}

Bivariate comparisons of characteristics of participants between the intensified and usual rehabilitation groups were examined using $\chi^{2}$-tests to compare categorical data and $t$-test to compare continuous data. ${ }^{35}$ All reported $P$-values were two-tailed, and $P<0.05$ was considered statistically significant.

We carried out multivariate logistic regressions of transitions to SNF including data from all study participants, and then carried out separate regressions using data from participants' scores on the Katz Index catego- ries. The mean multivariate-adjusted probabilities of transitions to SNF with corresponding 95\% confidence intervals (CI) were computed by adjusting for covariates. ${ }^{42}$ Adjusted odds ratios (OR) of transitions to SNF along with $95 \%$ CI were derived after adjusting for covariates. An OR $>1$ indicated that the probability of a transition to SNF for the intensified rehabilitation group was higher than that for the usual rehabilitation group.

\section{Sensitivity and multicollinearity}

Sensitivity analyses were used to explore alternative categories of ethnicity, (white people vs black people vs others), rehabilitation therapy type (threshold, $1.0 \mathrm{~h}$ per day) and Katz Index (0-2 vs 3-4 vs 5-6). Results for these alternative categories were similar to those of the original categories of ethnicity (white people vs non-white people), rehabilitation therapy type, (threshold, $0.5 \mathrm{~h}$ per day) and Katz Index scores (0-3 vs 4-5 vs 6), so the results for alternative categories are not included here. ${ }^{42,43}$

We also examined multicollinearity. ${ }^{41}$ A significant correlation between age and physical function at admission was found (coefficient $=0.75 ; P<0.001$, variance inflation factor $=4.61$.) For this reason, physical function at admission was retained and age was excluded from regressions. A significant correlation between delirium and severity of illness was found (coefficient $=0.79$; $P<0.001$, variance inflation factor $=4.14)$; severity of illness was retained and delirium was excluded from regressions. All models, both of overall participants and stratified participants by physical function at admission, fit well as determined by Hosmer-Lemeshow test results $(P=0.72$, overall participants; $P=0.85$, participants with no physical limitation; $P=0.63$, participants with mild physical limitations; $P=0.69$, participants with moderate to severe physical limitations.) All regressions had good discrimination as determined by $c$-statistics: $c=0.80$, overall participants; $c=0.83$, participants with no physical limitation; $c=0.68$, participants with mild physical limitations; $c=0.72$, participants with moderate to severe physical limitations. ${ }^{42,43}$ All statistics were carried out using SAS statistical software version 9.2 (SAS Institute, Cary, NC, USA).

\section{Results}

\section{Participant characteristics}

Of the total participants $(n=929)$, the numbers of participants of the intensified and usual rehabilitation therapy were 463 and 466, respectively. Table 1 presents participant characteristics by rehabilitation therapy type. Participant characteristics did not differ statistically between the intensified and usual rehabilitation therapy groups. 
Table 1 Participant characteristics by rehabilitation therapy type

\begin{tabular}{|c|c|c|c|c|}
\hline \multicolumn{2}{|c|}{$\%(n)$ or median (interquartile range) } & $\begin{array}{l}\text { Intensified } \\
\text { rehabilitation, } \\
n=463\end{array}$ & $\begin{array}{l}\text { Usual } \\
\text { rehabilitation, } \\
n=466\end{array}$ & ${ }^{\mp} P$ \\
\hline \multicolumn{2}{|l|}{ Age } & $80.4(73.8-89.8)$ & $81.3(74.5-91.6)$ & 0.08 \\
\hline \multicolumn{2}{|l|}{ Female } & $58(269)$ & $62(289)$ & 0.27 \\
\hline \multicolumn{2}{|l|}{ Non-white people } & $52(239)$ & $55(256)$ & 0.36 \\
\hline \multicolumn{2}{|l|}{ Living at risk } & $34(157)$ & $31(144)$ & 0.22 \\
\hline \multirow{3}{*}{$\begin{array}{l}\text { Severity of illness } \\
\quad \text { (APR-DRG) }\end{array}$} & Mild & $20(90)$ & $17(80)$ & \multirow[t]{3}{*}{0.14} \\
\hline & Moderate & 41 (189) & $40(187)$ & \\
\hline & Major & $39(184)$ & 43 (199) & \\
\hline Study site & Hospital B & 45 (209) & $43(201)$ & 0.66 \\
\hline \multirow{3}{*}{$\begin{array}{l}\text { Physical function } \\
\text { at admission }\end{array}$} & No limitation, Katz Index 6 & $44(203)$ & $41(191)$ & \multirow[t]{3}{*}{0.25} \\
\hline & Mild limitations, Katz Index 4-5 & 30 (139) & $28(131)$ & \\
\hline & Moderate-severe limitations, Katz Index $0-3$ & $26(121)$ & $31(144)$ & \\
\hline \multirow{2}{*}{\multicolumn{2}{|c|}{$\begin{array}{l}\text { Cognitive impairment before hospital admission } \\
\text { Delirium during hospitalization }\end{array}$}} & $24(113)$ & $27(126)$ & 0.23 \\
\hline & & $21(97)$ & $24(112)$ & 0.18 \\
\hline \multirow{8}{*}{$\begin{array}{l}\text { Principal diagnoses } \\
\text { at admission }\end{array}$} & Cardiovascular diseases & $17(79)$ & $16(74)$ & \multirow[t]{8}{*}{0.44} \\
\hline & Respiratory diseases & $14(64)$ & $14(66)$ & \\
\hline & Cancer and blood organ diseases & $9(42)$ & $8(37)$ & \\
\hline & Infectious diseases & $16(74)$ & $15(70)$ & \\
\hline & Endocrine/metabolic diseases & $13(59)$ & $12(56)$ & \\
\hline & Digestive/urogenital tract diseases & $15(69)$ & $16(74)$ & \\
\hline & Neurological diseases & $9(42)$ & $10(47)$ & \\
\hline & Others & $7(34)$ & $9(42)$ & \\
\hline
\end{tabular}

${ }^{\top} P$-values were derived from bivariate comparisons of participant characteristics by rehabilitation type. APR-DRG, all patient refined-diagnosis related group.

Table 2 Multivariate-adjusted probability of "transitions to skilled nursing facility" in overall participants and subsets by physical function at admission

\begin{tabular}{|c|c|c|c|c|}
\hline Mean ( $95 \%$ CI range) & $\begin{array}{l}\text { Intensified } \\
\text { rehabilitation } \\
n=463\end{array}$ & $\begin{array}{l}\text { Usual } \\
\text { rehabilitation } \\
n=466\end{array}$ & $\begin{array}{l}\text { †Odds ratio } \\
(95 \% \mathrm{CI})\end{array}$ & $P$ \\
\hline Overall participants, $n=929$ & $14 \%(8-22 \%)$ & $21 \%(15-29 \%)$ & $.59(.22-.96)$ & .02 \\
\hline $\begin{array}{l}\text { Subsets by physical function at admission } \\
\text { No physical limitation }\end{array}$ & $\begin{array}{l}9 \%(5-15 \%) \\
n=203\end{array}$ & $\begin{array}{l}11 \%(7-17 \%) \\
n=191\end{array}$ & $.91(0.63-1.20)$ & .53 \\
\hline $\begin{array}{l}\text { (Katz Index 6), } n=394 \\
\text { Mild physical limitations } \\
\text { (Katz Index } 4-5), n=270\end{array}$ & $\begin{array}{l}16 \%(12-21 \%) \\
n=139\end{array}$ & $\begin{array}{l}23 \%(19-28 \%) \\
n=131\end{array}$ & $.46(.17-.94)$ & .01 \\
\hline $\begin{array}{l}\text { Moderate to severe physical limitations } \\
\text { (Katz Index } 0-3), n=265\end{array}$ & $\begin{array}{l}18 \%(14-22 \%) \\
n=121\end{array}$ & $\begin{array}{l}28 \%(24-32 \%) \\
n=144\end{array}$ & $.34(.07-.89)$ & .004 \\
\hline
\end{tabular}

†Odds ratios were computed by adjusting for covariates. Odds ratio $>1$ indicated that the probability of intensified rehabilitation was higher than that of usual rehabilitation. CI, confidence intervals; SNF, skilled nursing facility.

\section{Multivariate-adjusted probability of "transitions to $S N F^{\prime \prime}$ in overall participants and subsets by physical function at admission}

Table 2 presents multivariate logistic regressions to determine the probability of transitions to SNF in the overall participants and subsets by physical function at admission. In the model of the overall participants $(n=929)$, the adjusted probability of transitions to SNF for participants in the intensified rehabilitation group (14\%; $95 \%$ CI $8-22 \%)$ was significantly lower than that for the usual rehabilitation group $(21 \%$; $95 \%$ CI 
15-29\%; OR 0.59; 95\% CI 0.22-0.96; $P=0.02$ ). In the model of participants with mild physical limitations (Katz Index 4-5, $n=270$ ), the adjusted probability of transitions to SNF for the intensified rehabilitation group (16\%; 95\% CI $12-21 \%)$ was significantly lower than that for the usual rehabilitation group $(23 \%$; $95 \%$ CI $19-28 \%$; OR $0.46 ; 95 \%$ CI $0.17-0.94 ; P=0.01)$. In the model of participants with moderate to severe physical limitations (Katz Index $0-3, n=265$ ), the adjusted probability of transitions to SNF for the intensified rehabilitation group (18\%; 95\% CI $14-22 \%)$ was significantly lower than that for the usual rehabilitation group (28\%; $95 \%$ CI $24-32 \%$; OR $0.34 ; 95 \%$ CI $0.07-$ $0.89 ; P=0.004)$. However, in the model of participants without physical limitation $(n=394)$, there was no significant difference in the adjusted probability of transitions to SNF between the intensified and usual rehabilitation groups $(P=0.53)$.

\section{Discussion}

The present findings provide new information about the effects of intensified rehabilitation on transition to SNF in community-living seniors with acute medical illness. There are notable reductions in the number of transitions to SNF by the intensified rehabilitation therapy group, and the magnitude of their effects becomes pronounced when their physical function at admission is more impaired. However, reductions in transitions to SNF by the intensified rehabilitation therapy group do not occur when their physical function is not impaired.

Hospital-associated frailty or disability occurs in more than one-third of hospitalized seniors, even when their medical conditions are successfully managed. ${ }^{2,3,7,8}$ Processes of hospital care - including numerous procedures, immobility and lack of adaptive accommodations - can lead to additional functional decline during hospitalization. ${ }^{7.8}$ Given the central role of hospitalizations in the disabling process, rehabilitation therapy has been studied as an effective method to manage hospitalassociated frailty or disability more effectively to promote restoration of function in interventions in the post-acute or intermediate care settings across diverse health systems. ${ }^{14-18}$ The present findings show that the reduction in transitions to SNF might stem from preventing or reducing hospital-associated frailty or disability by intensified rehabilitation, even at an acute care hospital setting.

According to a report from the Center for Medicare and Medicaid Services, Medicare charges for each transition from hospital to SNF were \$15 141 (daily rate $\$ 559)$ in 2010. ${ }^{31,32,44-46}$ Medicare charges for home healthcare per user were $\$ 5318$ (rate per visit \$145) in $2010 .^{37,44-46}$ Although no prior cost analysis study has evaluated a direct cost comparison between home health and SNF services as post-acute care, several lines of evidence lead to the expectation of cost-saving effects when hospitalized Medicare beneficiaries are transitioned to less expensive home health services instead of SNF services. ${ }^{44-46}$

Several implications for practice, policy and research can be derived from our findings. Although causality cannot be established by the present study, our findings have strong inferences that intensified rehabilitation therapy seemingly plays a "buffering" role in emancipating community-living seniors from transitions to SNF. ${ }^{11,17,47}$ This "buffering" role might make it more feasible for healthcare professionals to reprioritize rehabilitation therapy among the health issues of seniors with acute medical illness. Applying our findings to the agenda of public financial burdens, the positive relationship between the intensified rehabilitation at an acute care setting and reduction in transitions to SNF can have implications of saving public costs by reducing healthcare utilizations at a post-acute care setting. ${ }^{32,34,44-46}$ Given the magnified effects of rehabilitation therapy in subsets of more physically impaired seniors, further research of examining dosedependent effects of rehabilitation therapy on transitions to SNF in this group could be an important next step.

We acknowledge several limitations in data collection and study design. Because data collection was limited to two USA metropolitan hospitals, the findings of the present study cannot be generalized to other hospitals and health systems. The present study was a secondary analysis, and this limited the choice of covariates. Although physical function assessment at hospital admission relied on the same nursing staff, our analyses might have observer variation between hospital A and B. In addition, physical function was assessed once at hospital admission. This might not reflect the preadmission physical function accurately. Determining the intensity of rehabilitation in the present study is based on hospital LOS. This method might not entirely reflect the actual rehabilitation program and dosage across all disease entities. However, this method has been used by previous studies on rehabilitation outcomes. ${ }^{21,48}$ Because rehabilitation data are collected from administrative data, more specific rehabilitation program information (i.e. therapy modality, strength or frequency) is not included. Therefore, the present findings should be interpreted with caution and considered preliminary until they are confirmed in future studies with more representative data.

\section{Disclosure statement}

All authors declare no financial support or relationship that may pose conflicts of interest. 


\section{References}

1 Goodwin JS, Howrey B, Zhang DD, Kuo YF. Risk of continued institutionalization after hospitalization in older adults. J Gerontol A Biol Sci Med Sci 2011; 66A: 13211327.

2 Ferrucci L, Guralnik JM, Pahor M, Corti MC, Havik RJ. Hospital diagnoses, Medicare charges, and nursing home admissions in the year when older persons become severely disabled. JAMA 1997; 277: 728-734.

3 Fried LP, Ferrucci L, Darer J, Anderson G. Untangling the concepts of disability, frailty and comorbidity: implications for improved targeting and care. J Gerontol A Biol Sci Med Sci 2004; 59: 255-263.

4 Covinsky KE, Palmer RM, Fortinsky RH et al. Loss of independence in activities of daily living in older adults hospitalized with medical illnesses: increased vulnerability with age. J Am Geriatr Soc 2003; 51: 451-458.

5 Rudberg MA, Sager MA, Zhang J. Risk factors for nursing home use after hospitalization for medical illness. J Gerontol A Biol Sci Med Sci 1996; 51A: M189-M194.

6 Fortinsky RH, Covinsky KE, Palmer RM, Landerfeld CS. Effects of functional changes before and after during hospitalization on nursing home admission of older adults. J Gerontol A Biol Sci 1999; 54: M521-M526.

7 Hoenig HM, Rubenstein LZ. Hospital-associated deconditioning and dysfunction. J Am Geriatr Soc 1991; 39: 220 222.

8 Covinsky KE, Pierluissi E, Johnston CB. Hospitalizationassociated disability. JAMA 2011; 306: 1782-1793.

9 Howell S, Silberberg M, Quinn WV, Lucas JA. Determinants of remaining in the community after discharge: results from New Jersey's nursing home transition program. Gerontologist 2007; 47: 535-547.

10 Smith ER, Stevens AB. Predictors of discharges to a nursing home in a hospital-based cohort. J Am Med Dir Assoc 2009; 10: 623-629.

11 Mudge A, O'Rourke P, Denaro C. Timing and risk factors of functional changes associated with medical hospitalization in older adults. J Gerontol A Biol Sci Med Sci 2010; 65: 866-872.

12 Johnson M. Changing the culture of nursing homes: the physician's role. Arch Intern Med 2010; 170: 407-409.

13 Nolan M, Dellasega C. 'I really feel I've let him down': supporting family carers during long-term care placement for elders. J Adv Nurs 2000; 31: 759-767.

14 Kortebein P. Rehabilitation for hospital-associated deconditioning. Am J Phys Med Rehabil 2009; 88: 66-77.

15 Kortebein P, Bopp MM, Gragner CV et al. Outcomes of inpatient rehabilitation for older adults with debility. Am J Phys Med Rehab 2008; 87: 118-125.

16 Wells JL, Searbrook JA, Stolee P, Borrie MJ, Knoefel F. State of the art in geriatric rehabilitation. Part II: clinical challenges. Am J Phys Med Rehabil 2003; 84: 898-903.

17 Young J, Forster A, Green J, Bogle S. Post-acute transfer of older people to intermediate care services: the sooner the better? Age Ageing 2007; 36: 589-592.

18 Young J. The development of intermediate services in England. Arch Gerontol Geriatr 2009; 49 (Suppl 2): S21-S25.

19 Bachmann S, Finger C, Huss A et al. Inpatient rehabilitation specifically designed for geriatric patients: systematic review and meta-analysis of randomised controlled trials. BMJ 2010; 340: c1718.

20 Baztan JJ, Suarez-Garc1'a FM, Lopez-Arrieta J et al. Effectiveness of acute geriatric units on functional decline, living at home, and case fatality among older patients admitted to hospital for acute medical disorders: meta-analysis. BMJ 2009; 338: b50.

21 Jette DU, Warren RL, Wirtalla C. Relationship between therapy intensity and outcomes of rehabilitation in skilled nursing facilities. Arch Phys Med Rehabil 2005; 86: 373-379.

22 Lee WJ, Cheng YY, Liu CY et al. Dose-dependent effect of rehabilitation in functional recovery of older patients in the post-acute care unit. Arch Gerontol Geriatr 2012; 54: e290e293.

23 Landefeld CS, Palmer RM, Kresevic DM et al. A randomized trial of care in a hospital medical unit especially designed to improve the functional outcomes of acutely ill older patients. N Engl J Med 1995; 332: 1338-1344.

24 Reuben DB, Borok GM, Wolde-Tsadik G et al. A randomized trial of comprehensive geriatric assessment in the care of hospitalized patients. N EnglJ Med 1995; 332: 1345-1350.

25 Jayadevappa R, Chhatre S, Weiner M, Raziano D. Health resource utilization and medical care cost of acute care elderly unit patients. Value Health 2006; 9: 186-192.

26 Counsell S, Holder C, Liebenauer L et al. Effects of a multicomponent intervention on functional outcomes and process of care in hospitalized older patients: a randomized controlled trial of Acute Care for Elders (ACE) in a community hospital. J Am Geriatr Soc 2000; 48: 1572-1581.

27 Lee WJ, Peng LN, Cheng YY et al. Effectiveness of shortterm interdisciplinary intervention on postacute patients in Taiwan. J Am Med Dir Assoc 2011; 12: 29-32.

28 Chen LK, Chen YM, Hwang SJ et al. Effectiveness of community hospital-based post-acute care on functional recovery and 12-month mortality in older patients: a prospective cohort study. Ann Med 2010; 42: 630-636.

29 Arbaje AI, Maron DD, Yu Q et al. The geriatric floating interdisciplinary transition team. J Am Geriatr Soc 2010; 58: 364-370.

30 Yoo JW, Nakagawa S, Kim S. Delirium and transition to nursing home of hospitalized older adults: a controlled trial of assessing the interdisciplinary team-based "geriatric" care and care coordination by non-geriatrics specialist physicians. Geriatr Gerontol Int 2012; doi: 10.1111/j.14470594.2012.00905.x [Epub ahead of print].

31 Medicare Payment Advisory Commission (MedPAC). Skilled nursing facility services: assessing payment adequacy and updating payments. MedPAC Report to Congress. Washington, DC: Department of Health and Human Services, 2011; 147-171.

32 Centers for Medicare and Medicaid Services (CMS), Department of Health and Human Services. CMS-1410-F. Medicare program; Prospective payment systems and consolidated billing for skilled nursing facilities for fiscal year 2010. Fed Regist 2009; 74: 40288-40395.

33 Potetz L, Cubanski J, Neuman T. Medicare Spending and Financing: A Primer 2011 [Report \# 7731-03]. Menlo Park: The Henry J. Kaiser Family Foundation, 2011; 1-20.

34 Medicare Payment Advisory Commission (MedPAC). Hospital inpatient and outpatient services: assessing payment adequacy and updating payments. MedPAC Report to Congress. Washington, DC: Department of Health and Human Services, 2011; 38-66.

35 Stokes ME, Davis CS, Koch GG. Categorical Data Analysis Using the SAS System, 2nd edn. Cary, NC: SAS, 2009.

36 Kasper JD, Pezzin LE, Rice JB. Stability and changes in living arrangements: relationship to nursing home admission and timing of placement. J Gerontol B Psychol Sci Soc Sci 2010; 65: 783-791.

37 Averill RF, Goldfield NI, Muldoon J, Steinbeck BA, Grant TM. A closer look at all-patient refined DRGs. J AHIMA 2002; 73: 46-50. 
38 Katz S, Ford AB, Moskowitz RW, Jackson BA, Jaffe MW. Studies of illness in the aged: the index of ADL: a standardized measure of biological and psychosocial function. JAMA 1963; 185: 914-919.

39 Brorsson B, Asberg KH. Katz index of independence in ADL. Reliability and validity in short-term care. Scand $J$ Rehabil Med 1984; 16: 125-132.

40 Ciesla JR, Shi L, Stoskopf CH, Samuels ME. Reliability of Katz's Activities of Daily Living Scale when used in telephone interviews. Eval Health Prof 1993; 16: 190-203.

41 Inouye SK, van Dyck CH, Alessi CA et al. Clarifying confusion: the confusion assessment method: a new method for detection of delirium. Ann Intern Med 1990; 113: 941-948.

42 Hosmer DW, Lemeshow S. Applied Logistic Regression, 2nd edn. Hoboken, NJ: John Wiley \& Sons, Inc, 2005.

43 Harrell FE Jr, Lee KL, Mark DB. Multivariable prognostic models: issues in developing models, evaluating assumptions and adequacy, and measuring and reducing errors. Stat Med 1996; 15: 361-387.

44 Center for Medicare and Medicaid Services. Medicare and Medicaid Statistical Supplement. 2011 Edition. Table 6.2.
Covered admissions, covered days of care, covered charges, and program payments for skilled nursing facility services used by Medicare beneficiaries, by demographic characteristics, type of entitlement, and discharge Status: calendar year 2010. [Cited 3 Jun 2012.].

45 Center for Medicare and Medicaid Services. Medicare and Medicaid Statistical Supplement. 2011 Edition. Table 7.2. Persons served, visits, total charges, visit Charges, and program payments for Medicare home health agency services, by demographic characteristics: calendar year 2010 . [Cited 3 Jun 2012.].

46 National Association for Home Health and Hospice. Basic statistics about home care 2010. [Cited 3 Jun 2012.].

47 Gill TM, Gahbauer EA, Han L, Allore HG. The relationship between intervening hospitalizations and transitions between frailty states. J Gerontol A Biol Sci Med Sci 2011; 66: 1238-1243.

48 Hoenig H, Rubenstein LV, Sloane R et al. What is the role of timing in the surgical and rehabilitative care of community-dwelling older persons with acute hip fracture? Arch Intern Med 1997; 157: 513-520. 